\title{
Turbulence Calculations of a Dual-Structure Oxygen Lance for Converter Based on Hydrodynamics
}

\author{
Hongbin Jia, ${ }^{1,2}$ Yanxin Li, ${ }^{1}$ Peng Han $\mathbb{D}^{1},{ }^{1}$ Kun Liu $\left(\mathbb{D},{ }^{1}\right.$ Haolei Han, ${ }^{1}$ and Lianghua Feng ${ }^{1}$ \\ ${ }^{1}$ School of Materials and Metallurgy, University of Science and Technology Liaoning, Anshan 114051, China \\ ${ }^{2}$ Iron and Steel Research Institute, Ansteel Group, Anshan 114009, China \\ Correspondence should be addressed to Peng Han; hanpeng@ustl.edu.cn and Kun Liu; liukun0206@sina.com
}

Received 28 June 2021; Revised 20 August 2021; Accepted 14 September 2021; Published 8 October 2021

Academic Editor: Adnan Maqsood

Copyright (c) 2021 Hongbin Jia et al. This is an open access article distributed under the Creative Commons Attribution License, which permits unrestricted use, distribution, and reproduction in any medium, provided the original work is properly cited.

The oxygen lance is a piece of special equipment in the converter steelmaking process for blowing oxygen into the molten steel. After more than 80 years of development, the structure and function of the oxygen lance have undergone many changes. In this paper, based on the theory of hydrodynamics, the jet behavior characteristics of a dual-structure oxygen lance for the converter are determined and optimized by CFD simulations and compared with those of the traditional-structure oxygen lance. The research results show that multiple jets deflect to the central axis of the oxygen lance during movement and the inclination angle of the nozzle holes influences the jet deflection. A decrease in the nozzle hole angle results in an increase in the mutual suction between the streams. With the increasing flow rate through the large holes in the new dual-structure oxygen lance, the dynamic radial pressure increases at the middle of the jet. The jet flow characteristics of the new dual-structure oxygen lance are better than those of the traditional oxygen lance. Its impact on the molten pool includes greater momentum, a larger impact area, and a more uniform and powerful stirring of the molten pool. A nozzle angle of $14^{\circ}$ combined with a flow rate ratio of $65 \%$ and a nozzle angle of $17^{\circ}$ combined with a flow rate ratio of $35 \%$ are the optimal parameters for the new dual-structure oxygen lance.

\section{Introduction}

The oxygen lance is an essential piece of equipment in the converter steelmaking process and has been continuously developed since the invention of this process. As the continuous increase of the converter capacity requires larger amounts of oxygen for the smelting reaction, considerable research on the optimization of the oxygen lance structure has been conducted [1-3]. The converter oxygen lance has evolved from a structure with a single hole to three holes and to the current five holes and six holes $[4,5]$. The development of oxygen lances includes not only the increase in the number of nozzle holes but also the continuous changes in their diameter and inclination angle to adapt to converters with increasing capacity [6-8]. Cao et al. [9] investigated the highly complex interaction process inside an $80 \mathrm{t}$ converter by a cold model experiment and the corresponding numerical simulation. Nordquist et al. $[10,11]$ focused their research on the top-blown water model and demonstrated that the nozzle diameter, lance height, aspect ratio, and gas flow rate influence the penetration depth and the swirl phenomena. Liu et al. [12] investigated the jet behavior of interlaced multiple nozzles and the impingement of multiple jets onto the surface of the molten metal bath in a steelmaking converter. The interlaced jet hydrodynamic behaviors such as jet profile, coalescence characteristics, dynamic parameter distributions, penetration depth, and impact area, as well as the time-dependent evolution of the liquid bath are discussed. Wang and Jia et al. $[13,14]$ investigated the dynamic characteristics of supersonic doubleparameter jets and their interaction with the molten pool. The effects of jet inclination angles and gas flow ratios on gas intensity, coalescence characteristics, and dynamic parameter distributions were discussed. Problems of the currently used traditional-structure oxygen lances include insufficient stirring capacity and low oxygen-supply ability. It has become increasingly difficult to change the traditional oxygen lance structure to optimize the jet behavior and improve the 
steelmaking process. To address these problems, based on the theory of hydrodynamics, the jet behavior characteristics of the dual-structure oxygen lance for the converter are determined and optimized by CFD simulations and compared with those of the traditional-structure oxygen lance. The method of optimizing jet behavior characteristics by changing structural parameters can provide directions for the development of converter oxygen lances.

\section{Simulation Method and Conditions}

2.1. Geometric Model and Simulation of the Oxygen Lance. Based on the structural parameters of the traditional fivehole oxygen lance for the 260-t converter industry and the traditional six-hole oxygen lance, a novel dual-structure sixhole oxygen lance nozzle is designed. The two structures are characterized by different flow rates, inclination angles, and center distance designs. The nozzle holes are staggered. The nozzle structure of the oxygen lance is shown in Figure 1. In this figure, $D$ represents the outer diameter of the oxygen lance, $\alpha_{1}$ and $\alpha_{2}$ denote the inclination angle between the large and small holes and the central axis, respectively, $L 1$ and $L 2$ correspond to the distance between the large and small holes and the central axis, respectively, $d_{11}$ and $d_{21}$ represent the inlet diameters of the large and small holes, $d_{12}$ and $d_{22}$ denote the throat diameters of the large and small holes, and $d_{13}$ and $d_{23}$ correspond to the exit diameter of the large and small holes. The specific size parameters are shown in Table 1 . Cases 1 to 8 refer to the dual-structure oxygen lance nozzle, and cases 9 and 10 to the traditional-structure oxygen lance nozzle. The identifier of a dual-structure oxygen lance nozzle consists of the values for the large-hole angle, small-hole angle, and the proportion of large-hole flow, while the identifier of a traditional-structure oxygen lance nozzle contains the value of the angle and the number of spray holes.

2.2. Geometric Modeling and Mesh Generation. In this work, the oxygen lance nozzle was modeled based on the Cartesian coordinate system. Since the nozzles are distributed symmetrically, the calculation domain was set to consider only one-third of the entire model for the six-hole oxygen lance and one-fifth of the entire model for the five-hole oxygen lance to simplify the calculation and increase the convergence speed [15-18]; the model mainly includes two Laval nozzles and a sufficiently large space. The latter consists of a segment of one-third of a cylinder with a radius of $1.2 \mathrm{~m}$ and a height of $3 \mathrm{~m}$. The geometric model is shown in Figure 2.

In this study, CAE preprocessing software ICEM CFD 15.0 developed by the company ANSYS is used for mesh generation. A hexahedral-structured grid was used, and the calculation area consisted of an oxygen lance nozzle and an infinite space. Furthermore, the grid was encrypted for the area where the speed changes greatly (the inside of the oxygen lance nozzle and the nozzle outlet area), and the minimum mesh quality was set to 0.75 ; the number of mesh elements was about 3 million. The main view of the mesh model and a partially enlarged view of the grid are shown in Figure 3.

\subsection{Implementation of the Mathematical Model}

2.3.1. Basic Assumptions. The CFD software ANSYS Fluent 15.0 is used in the numerical calculations, the ideal gas option is used, and the periodic boundary conditions are set. In the calculation of the jet flow, the vortex effect can be neglected. The gas source is oxygen, which is an ideal compressible gas suitable for the application of the equation of state of an ideal gas. The internal connection of the Laval nozzle is smooth. The entire flow process is a three-dimensional, steady-state, compressible, and nonisothermal process.

2.3.2. Governing Equations. The mass conservation equation can be expressed as

$$
\frac{\partial\left(\rho u_{i}\right)}{\partial x_{i}}=0
$$

where $\rho$ is the fluid density, $\mathrm{kg} / \mathrm{m}^{3}$, and $u_{\mathrm{i}}$ is the velocity in direction $i, \mathrm{~m} / \mathrm{s}$.

The momentum conservation equation in direction $i$ can be expressed as follows:

$$
\begin{aligned}
\frac{\partial\left(\rho u_{i} u_{j}\right)}{\partial x_{i}} & =-\frac{\partial \rho}{\partial x_{i}}+\frac{\partial\left(\tau_{i j}-\rho \overline{u_{i} u_{j}}\right)}{\partial x_{j}} \\
\tau_{i j} & =\mu\left(\frac{\partial u_{j}}{\partial x_{i}}+\frac{\partial u_{i}}{\partial x_{j}}-\frac{2 \partial u_{k}}{3 \partial x_{k}} \delta_{i j}\right)
\end{aligned}
$$

where $p$ is the pressure, $\mathrm{Pa}, \tau_{\mathrm{ij}}$ is the viscosity stress caused by the molecular viscosity, $\mathrm{N} / \mathrm{m}^{2}, \delta_{\mathrm{ij}}$ is the Kronecker function (if $i=j, \delta_{\mathrm{ij}}=1$; if $i \neq j, \delta_{\mathrm{ij}}=0$ ), and $\mu$ is the viscosity coefficient, $\mathrm{Pa} \cdot \mathrm{s}$.

According to the Boussinesq approximation, the Reynolds stress term in the momentum equation $-\rho \overline{u_{i} u_{j}}$ represents the effect of turbulence:

$$
-\rho \overline{u_{i} u_{j}}=\mu_{t}\left(\frac{\partial u_{j}}{\partial x_{i}}+\frac{\partial u_{i}}{\partial x_{j}}\right)-\frac{2}{3}\left(\rho k+\mu_{t} \frac{\partial u_{k}}{\partial x_{k}}\right) \cdot \delta_{i j}
$$

In the above equation, $\mu_{\mathrm{t}}$ is the turbulent viscosity coefficient, $\mathrm{Pa} \cdot \mathrm{s}$, and $k$ is the turbulent kinetic energy, $\mathrm{J} / \mathrm{kg}$.

The energy conservation equation can be expressed as

$$
\frac{\partial\left[u_{i}\left(\rho C_{p} T+p\right)\right]}{\partial x_{i}}=-\frac{\partial}{\partial x_{i}}\left(\lambda \frac{\partial T}{\partial x_{i}}+\frac{C_{p} \mu_{t}}{\operatorname{Pr}_{t}} \frac{\partial T}{\partial x_{i}}\right)+\frac{\partial}{\partial x_{i}}\left(\tau_{i j} u_{j}-u_{j} \rho \overline{u_{i} u_{j}}\right)
$$

where $T$ is the temperature, $\mathrm{K}, C_{\mathrm{p}}$ is the specific heat capacity, $\mathrm{J} /(\mathrm{kg} \cdot \mathrm{K}), \lambda$ is the thermal conductivity, $\mathrm{W} /(\mathrm{m} \cdot \mathrm{K})$, and $\operatorname{Pr}_{\mathrm{t}}$ is the Prandtl number of the energy, which was here set to 0.85 .

2.3.3. Turbulence Model. The standard $k$-E model was proposed by Lauder and Spalding and consists of the turbulent kinetic energy transport equation and dissipation rate 


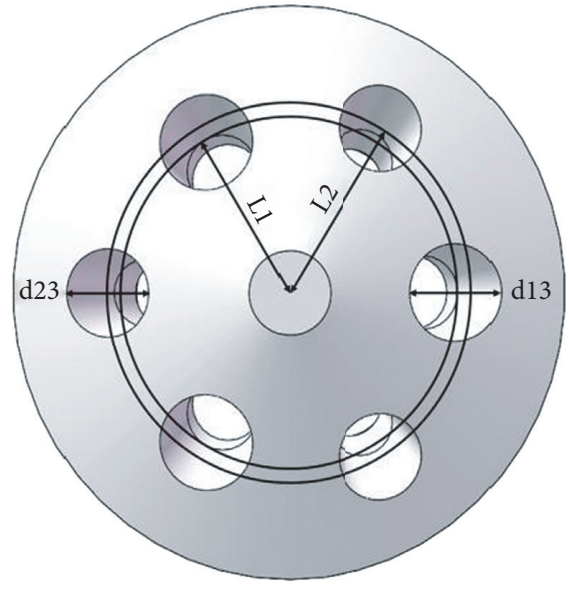

(a)

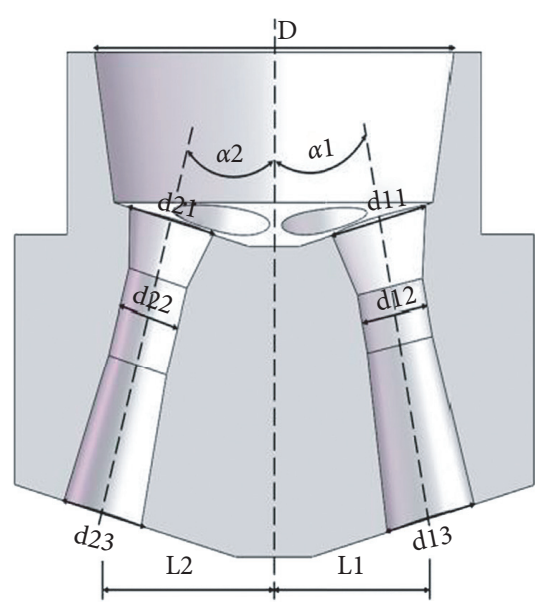

(b)

Figure 1: Schematic diagram of the dual-structure oxygen lance nozzle structure. (a) Top view of the oxygen lance nozzle. (b) Profile of the oxygen lance nozzle.

equation. This model does not require excessive computer memory resources and has a short calculation time and good accuracy. For these reasons, the $k-\varepsilon$ model has become the most widely used turbulence model in engineering. The standard $k-\varepsilon$ model equations are as follows:

$$
\begin{aligned}
& \frac{\partial\left(\rho k u_{i}\right)}{\partial x_{i}}=\frac{\partial}{\partial x_{j}}\left[\left(\mu+\frac{\mu_{i}}{\alpha_{k}}\right) \frac{\partial k}{\partial x_{j}}\right]+G_{k}+G_{b}-\rho \varepsilon-Y_{M}+S_{k} \\
& \frac{\partial\left(\rho \varepsilon u_{i}\right)}{\partial x_{i}}=\frac{\partial}{\partial x_{j}}\left[\left(\mu+\frac{\mu_{i}}{\alpha_{\varepsilon}}\right) \frac{\partial \varepsilon}{\partial x_{j}}\right]+C_{1 \varepsilon} \frac{\varepsilon}{k}\left(G_{k}+C_{3 \varepsilon} G_{b}\right)-C_{2 \varepsilon} \rho \frac{\varepsilon^{2}}{k}+S_{\varepsilon} .
\end{aligned}
$$

In these above equations, $G_{\mathrm{k}}$ and $G_{\mathrm{b}}$ are the turbulent kinetic energy generation terms caused by the average velocity gradient and buoyancy, respectively, YM is the contribution of the wave expansion to the total dissipation rate in compressible turbulence, $C_{1 \varepsilon}, C_{2 \varepsilon}$, and $C_{3 \varepsilon}$ are empirical constants with values of $1.44,1.92$, and 1 , respectively, $\sigma_{\mathrm{k}}$ and $\sigma_{\varepsilon}$ are the Prandtl numbers corresponding to the turbulent kinetic energy $k$ and dissipation rate $e$ with values of 1.0 and 1.3 , respectively, and $S_{\mathrm{k}}$ and $S_{\varepsilon}$ are user-defined source items. The turbulent viscosity coefficient $\mu$ is calculated according to

$$
\mu=\rho C_{\mu} \frac{k^{2}}{\varepsilon},
$$

where $C_{\mu}$ is a constant that is determined via the basic turbulence experiment; its default value is 0.09 .

2.3.4. Boundary Conditions. All boundary conditions are selected with reference to actual converter production conditions. The inlet boundary is set as the pressure inlet, the outlet boundary is set as the pressure outlet, and the symmetrical plane uses symmetrical boundary conditions [19]. The upper-end surface of the nozzle hole of the oxygen lance is set as the pressure inlet, and the gas used is oxygen, which is assumed to be a compressible ideal gas. The wall of the Laval tube is set as the wall surface, using standard wall functions $[20,21]$, and the other outer surfaces are set as pressure outlets. The boundary conditions and operating parameters are shown in Figure 4 and Table 2.

2.4. Grid Independence Verification. The density of the calculation grid has a great influence on the accuracy and precision of the numerical results. Therefore, to test the sensitivity of the calculation results on the grid, the velocity distribution along the central axis of the oxygen lance jet was compared and analyzed for five different numbers of grid elements of the mathematical model, as shown in Figure 5. The oxygen lance position refers to the distance between the oxygen lance nozzle and the liquid level of the molten pool. In theoretical analysis, it is usually expressed by the ratio of the oxygen lance position to the exit diameter of the nozzle, represented by $H$ in this paper. The results show that, as the number of grid elements in the computational domain increases, the computational accuracy gradually improves. Beyond 3 million, the number of grid elements has little effect on the calculation results. Consequently, the grid element number of 2991748 is most suitable for the numerical simulations. 


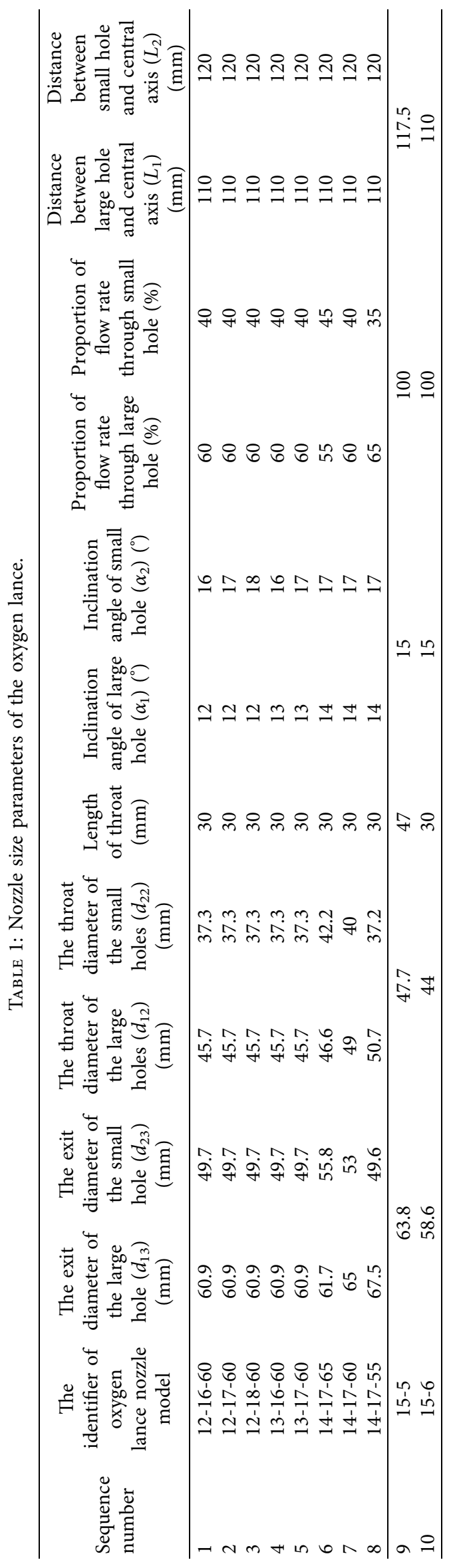




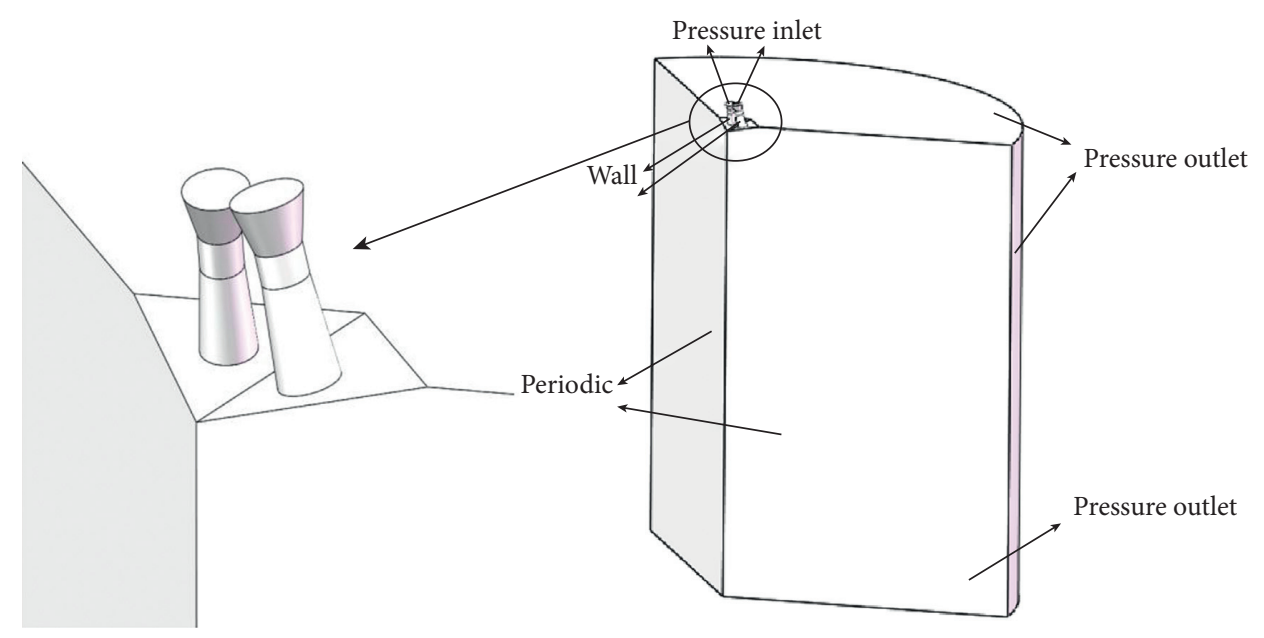

Figure 2: Physical model and partially enlarged view of the nozzle.

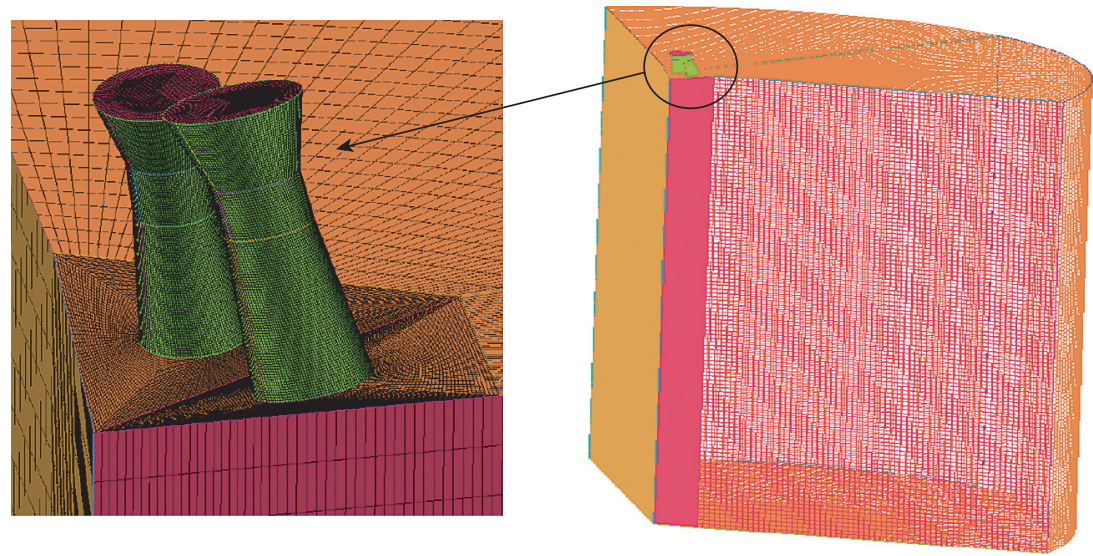

Figure 3: Main view of the grid model and partially enlarged view of the nozzle grid.

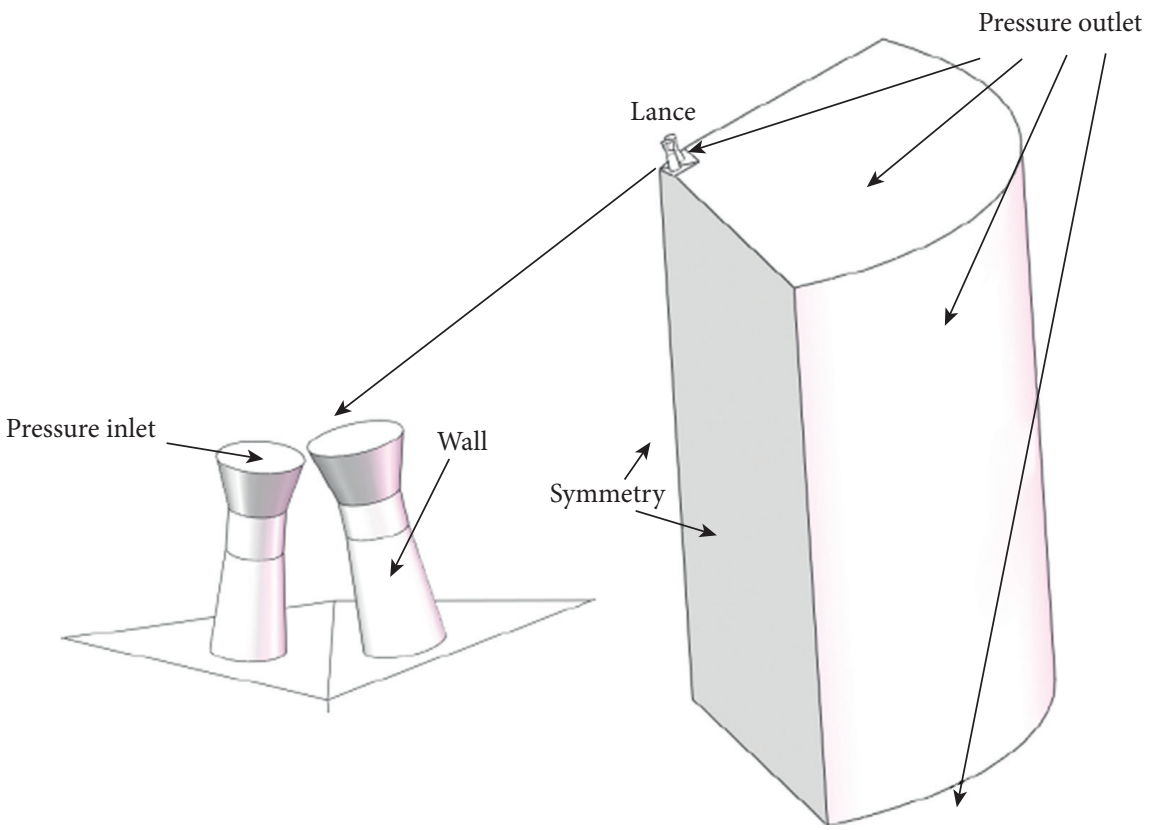

FIGURE 4: Schematic diagram of the boundary conditions. 
TABLE 2: Operating parameters.

\begin{tabular}{lc}
\hline Boundary condition & Value \\
\hline Environmental pressure $P_{b}, \mathrm{MPa}$ & 0.104 \\
Environmental temperature $T_{b}, \mathrm{~K}$ & 290 \\
Design pressure $P_{0}, \mathrm{MPa}$ & 0.97 \\
Inlet temperature $T_{0}, \mathrm{~K}$ & 290 \\
\hline
\end{tabular}

\section{Results and Discussion}

3.1. Influence of Nozzle Angle on Radial Distance of the Jet. The schematic diagram of the jet is shown in Figure 6. Each jet deviates from the central line of the respective nozzle and deflects to the central axis of the oxygen lance nozzle. As an explanation for these results, during the movement of a single jet, the jet will continue to spread, exchange energy and mass in the boundary layer, and swirl the surrounding media, which leads to the continuous expansion of the jet shape and continuous decrease in velocity. However, multiple jets draw the medium from the central axis of the oxygen lance during the movement, which causes them to deflect to the central axis of the oxygen lance. After a certain distance of movement, the deviation of the jet stream from the central axis of the original nozzle hole increases continuously, and the radial distance tends to stabilize. As the oxygen lance position continues to rise, the jet streams will gradually merge, and multiple jets will eventually become one jet.

The variation of the radial distance of the large hole and small-hole jet with the oxygen lance position is displayed in Figure 7. Referring to Figures 7(a) and 7(b), the change of the inclination angle of the small hole has a significant effect on the radial distance of the small-hole jet but only little effect on that of the large-hole jet. With decreasing hole angle, the radial distance and the distance between jet and the central axis of the oxygen lance decrease. As evident from Figures $7(\mathrm{c})$ and $7(\mathrm{~d})$, the change in the inclination angle of the large hole affects the radial distance of the large-hole jet significantly but influences that of the small-hole jet only to a small extent. A decrease in the nozzle angle will result in a decrease in the radial distance of the jet because the nozzle angle significantly contributes to the radial distance of the jet. If the jet moves closer to the central axis of the oxygen lance, the mutual drawing effect between the jets increases. When the radial distance ceases to change significantly, the streams have been fused. As the nozzle angle increases, the fusion distance and radial distance of the jet increase.

3.2. Influence of Nozzle Angle on the Jet Impact Area. Cloud diagrams of the effective impact area for the two new dual-structure oxygen lances and the traditional five-hole oxygen lance at different oxygen lance positions are displayed in Figure 8. The degree of fusion of each stream and the growth of the effective impact area are clearly visible in these diagrams. The fusion distance is the oxygen lance position with maximum radial distance. The jet cloud diagrams for the oxygen lance positions indicate that the streams have been fused when the radial distance assumes the maximum value. The fusion distance can be used to judge the independent state of each jet, which improves with increasing fusion distance. The jets of the 12-16-60 oxygen lance at the oxygen lance position of 15 De begin to merge; at the same position, the 12-18-60 oxygen lance still maintains good jet independence, but the jets start to merge at an oxygen lance position of $20 \mathrm{De}$. For the traditional five-hole oxygen lance, the jets start to merge at the oxygen lance position of $30 \mathrm{De}$. With the increase in the oxygen lance position, the effective impact area of each lance gradually increases due to the diffusion characteristics of the free jet. However, the effective impact area of the 12-18-60 oxygen lance is larger than that of the 12-16-60 lance since increasing the angle of the nozzle holes reduces the drawing effect between the jets and increases the radial distance, which is conducive to maintaining the independence of the jets, thereby increasing the effective impact area.

The effective impact area is calculated by using the CFD software (ANSYS Fluent15.0); then, the data of the effective impact area is selected for postprocessing by using the Tecplot software and presented in the format of pictures. Finally, the specific value of the effective impact area is obtained by using image processing software. Under the condition that the flow rate ratio through the large holes is $60 \%$, the effect of the large hole and small hole angle changes on the effective impact area, fusion distance, and radial distance is shown in Figure 9. While increasing the angle of the large and small holes can increase the effective impact area, the angle of the small hole exerts a greater impact. The numerical simulation results are consistent with the original design concept. For the dual-structure oxygen lance, the effective impact area of the 14-17-60 oxygen lance is the largest, but it is slightly smaller than that of the traditional five-hole oxygen lance because its nozzle hole angle is $15^{\circ}$ and the distance between each nozzle hole and the central axis of the nozzle is the largest. With increasing radial distance, both the fusion distance and the effective impact area increase because the effective impact area of the jet is related to the independence of each jet. At greater radial distance, the independence of the jet improves, the fusion between the streams slows down, and the effective impact area increases.

3.3. Influence of Flow Rate on the Distribution of Axial Jet Parameters. The dynamic axial parameter distribution of the oxygen lance jet in the steelmaking converter affects the interaction process between the jet and the molten pool. Especially the attenuation characteristics of the axial velocity and dynamic pressure of the jet determine its impact force when it hits the surface of the molten pool. It further affects the pit shape and stirring effect caused by the jet impact on the molten pool. The diagrams in Figures 10-13 reflect the changes of velocity, density, temperature, and dynamic pressure on the centerline of the jet with the oxygen lance position. The results show that, with the axial flow of the jet, the trend of the change in parameters on the jet centerline (velocity, density, temperature, and dynamic pressure) is basically the same for the traditional and the dual-structure 


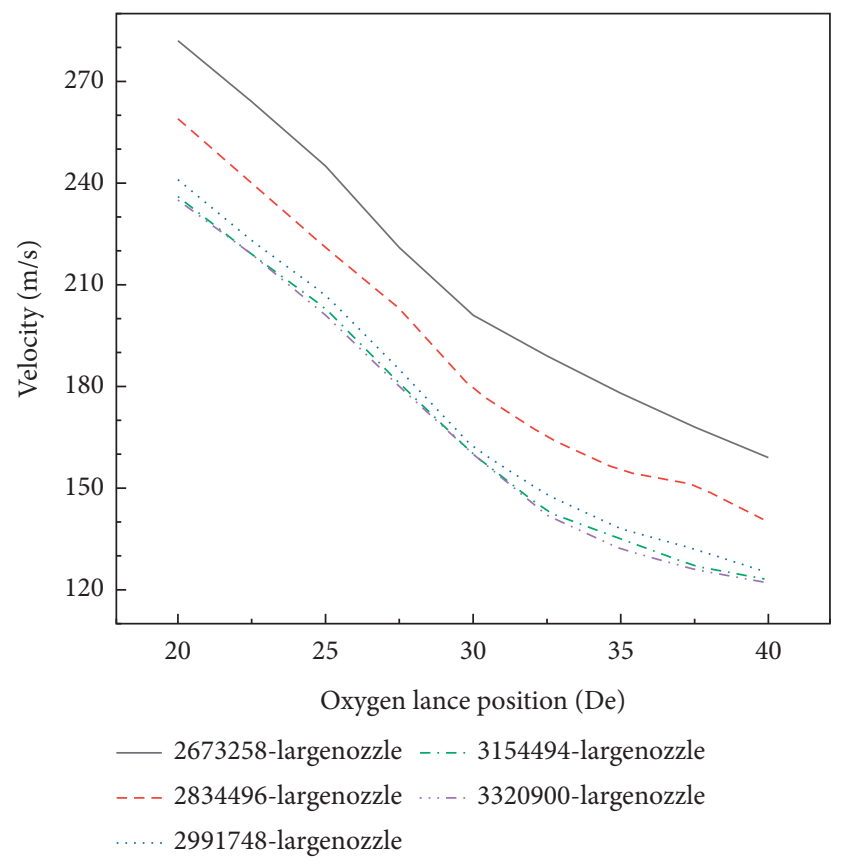

(a)

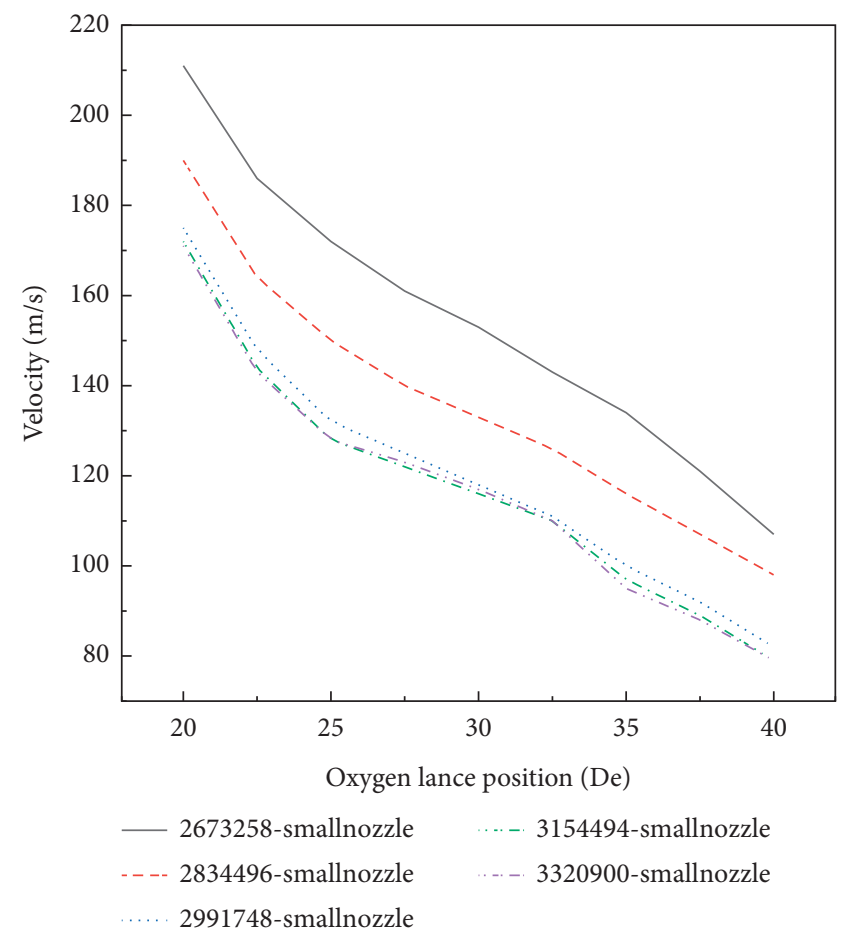

(b)

FIGURE 5: Velocity distribution of the central jet axis for different numbers of computational domain grid elements. (a) Velocity distribution for the large hole jet. (b) Velocity distribution for the small hole jet.

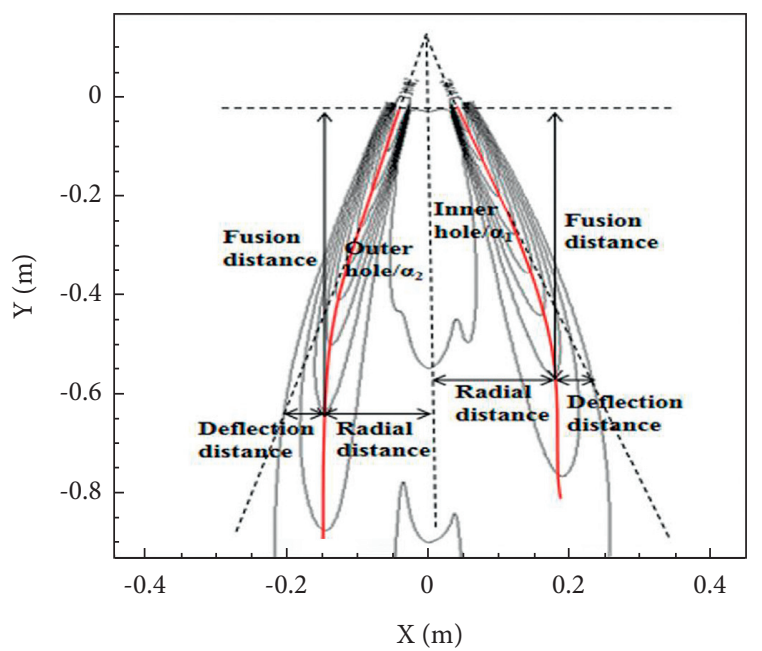

FIgURE 6: Schematic diagram of the jet.

oxygen lance. The velocity, density, and dynamic pressure gradually decrease, and the temperature of the jet will gradually increase due to the heat exchange with the environment. For the dual-structure oxygen lance, the jet characteristics of the large nozzle hole are better than those of the traditional oxygen lance, and with increasing nozzle flow rate, the jet velocity, density, and dynamic pressure increase while the jet temperature decreases. The specific design of the dual-structure oxygen lance creates an inconsistency by the simultaneous presence of large and small holes, whereby the large-hole oxygen flow accounts for $55-65 \%$ of the total oxygen flow. The superior behavior of this oxygen lance can be attributed to its specific design, where the large-hole jet is surrounded by the jet flow of the small holes to ease the attenuation speed of the jet flow of the large hole and improve its jet strength. When the oxygen lance is positioned beyond $30 \mathrm{De}$, the further decrease in jet velocity, density, temperature, and dynamic pressure diminishes, the gap between the various lances is small, and the stirring effect of the jet impacting the molten pool is stable, which can ensure the smelting requirements.

The length of the supersonic region refers to the distance from the nozzle outlet to the jet velocity attenuation to sonic velocity [22], which can intuitively describe the decay rate of the supersonic jet. By comparing the velocity distribution of the traditional and the dual-structure oxygen lance on the nozzle axis, it can be concluded that the length of the supersonic region decreases in the order: large hole of the dualstructure oxygen lance, traditional five-hole oxygen lance, traditional six-hole oxygen lance, and small hole of the dualstructure oxygen lance; furthermore, the length of the supersonic zone increases with increasing nozzle flow rate in the dual-structure oxygen lance.

The velocity and static pressure distribution on the geometric central axis of the nozzle of the porous oxygen lance can indicate the time and degree of mixing of multiple jets at the boundary [23]. Figure 14 shows the variation of the dynamic parameters on the central oxygen lance axis for each simulated lance with the oxygen lance position. As indicated by the results, the dynamic parameters on the 


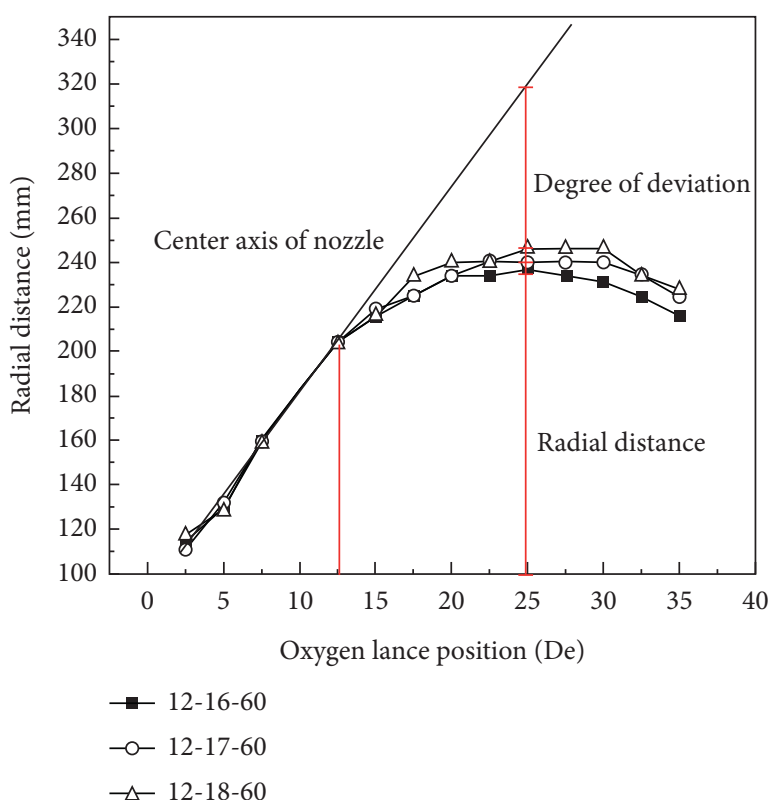

(a)

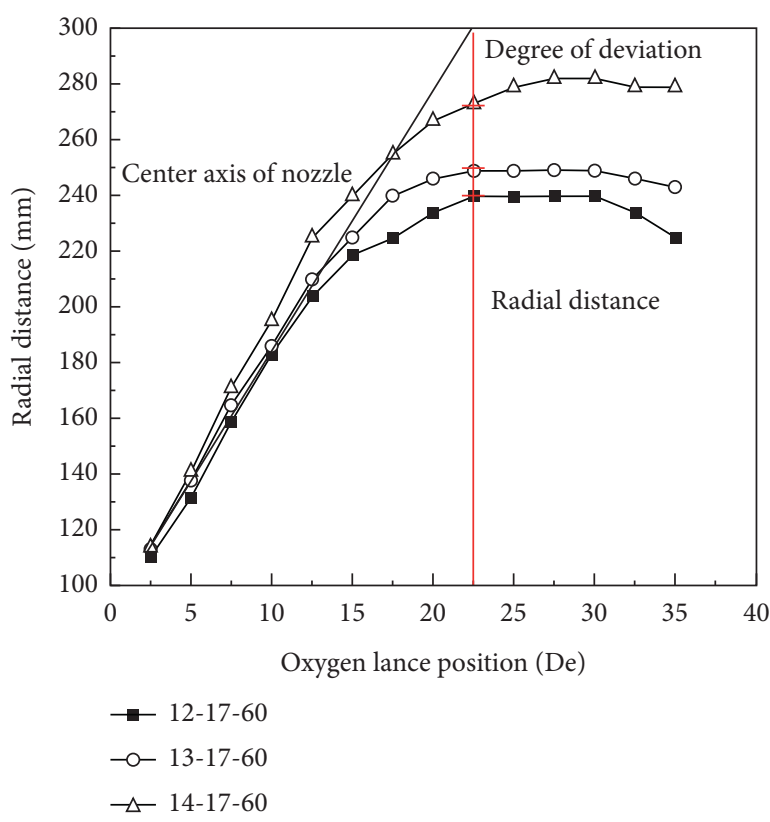

(c)

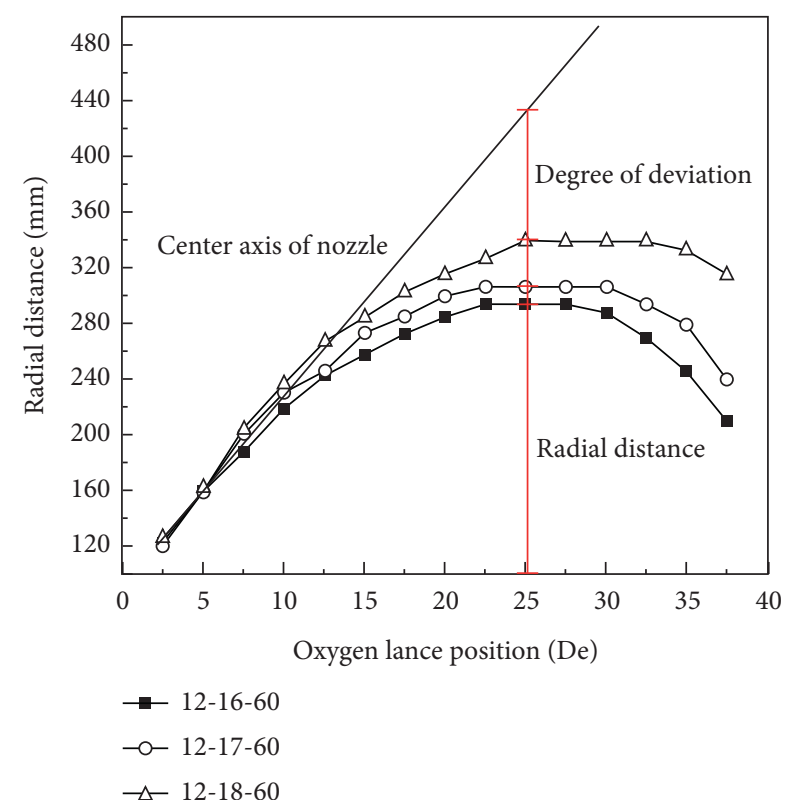

(b)

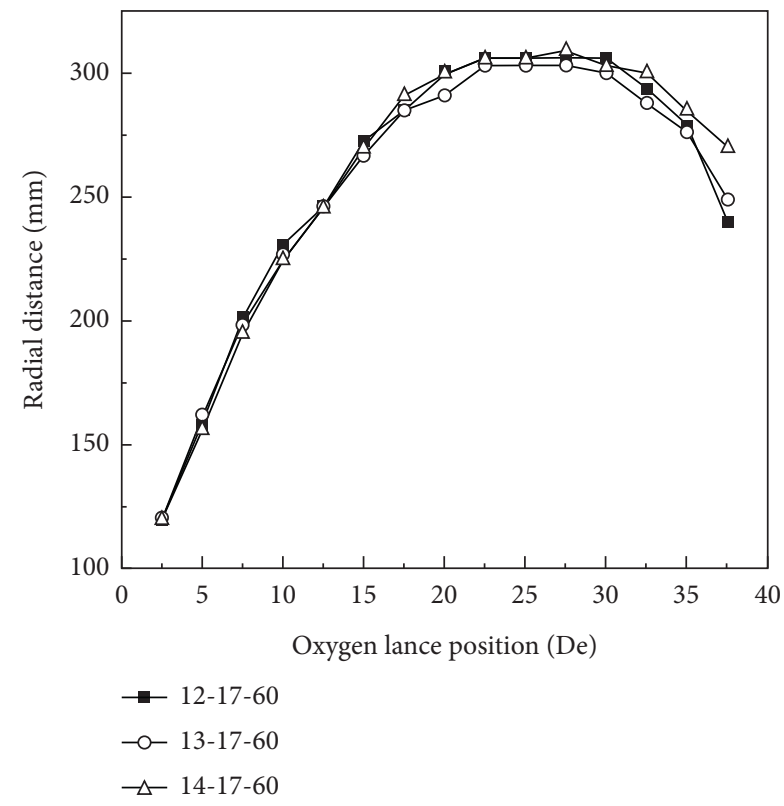

(d)

Figure 7: Variation of the radial distance of the large hole and small-hole jet with the oxygen lance position. (a) Radial distance of the largehole jet. (b) Radial distance of the small-hole jet. (c) Radial distance of the large-hole jet. (d) Radial distance of the small-hole jet.

central axis of the dual-structure oxygen lance do not differ significantly for the three employed flow rate ratios. However, when the oxygen lance is positioned further than $15 \mathrm{De}$ from the nozzle outlet, the jet velocity on the central axis is significantly greater for the dual-structure oxygen lance than for the traditional oxygen lance.

As shown in Figure 14(a), the jet forms a negative pressure zone near the central axis of the oxygen lance, which can be explained by surrounding gas being entrained by the jet, causing the pressure to be lower inside the jet than outside the jet. Under the effect of this pressure difference, the jet deviates from the axis of the jet hole and tends to converge. The position of the negative pressure area of the nozzle of the dual-structure oxygen lance is farther from the nozzle outlet than for the traditional oxygen lance nozzle. In the actual smelting process, a greater distance of the negative pressure area from the nozzle outlet results in higher effectiveness in the reduction of sticking guns, burning guns, etc., and in increased service time of the oxygen lance. As shown in Figure 14(b), as the axial distance from the nozzle outlet increases, the velocity on the geometric central axis of the oxygen lance nozzle gradually increases. However, if the 

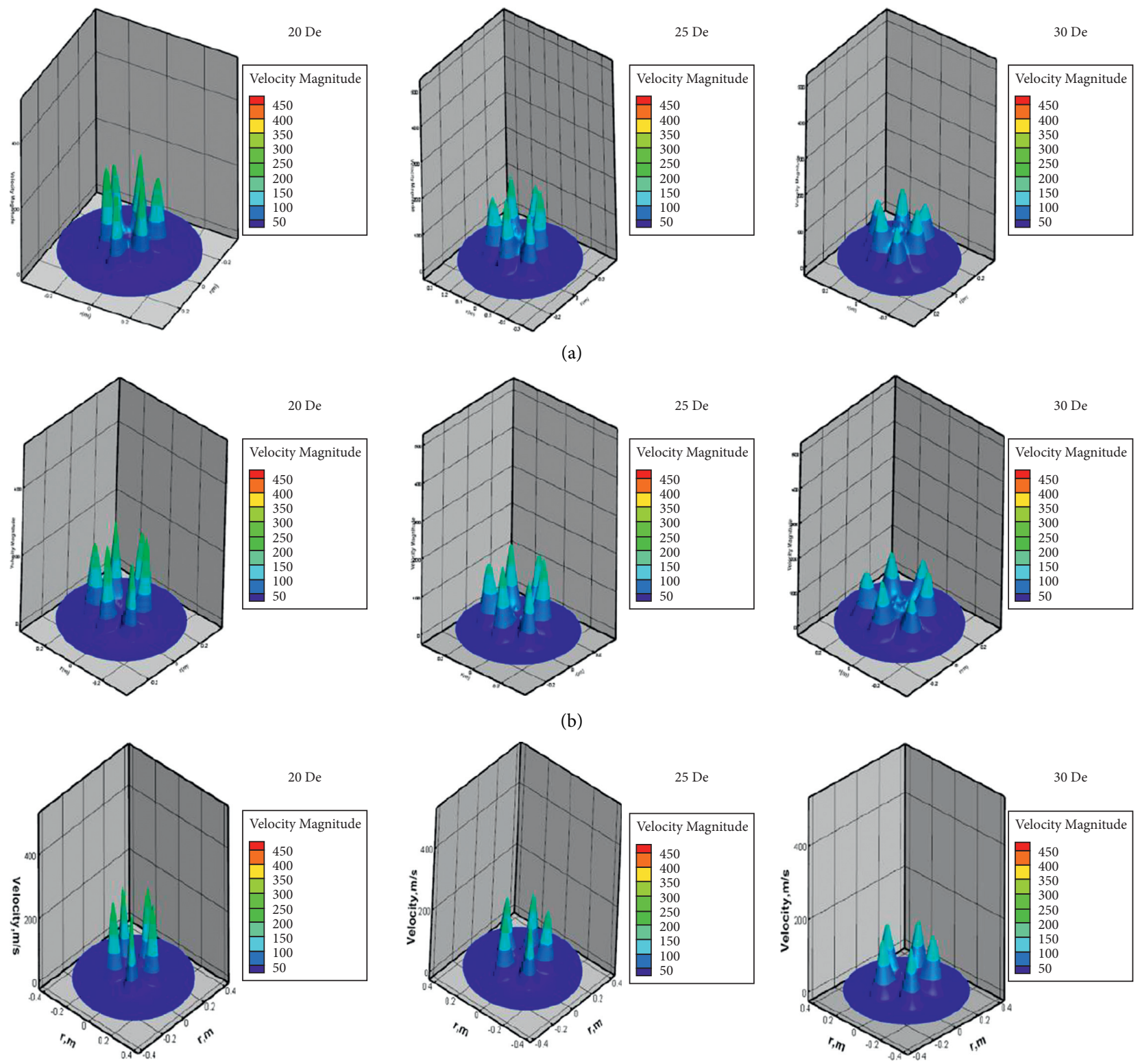

(c)

Figure 8: Cloud diagram of the effective impact area for the different oxygen lances at different oxygen lance positions. (a) Cloud diagram of the effective impact area for the 12-16-60 oxygen lance. (b) Cloud diagram of the effective impact area for the 12-18-60 oxygen lance. (c) Cloud diagram of the effective impact area for the 15-5 oxygen lance.

oxygen lance position is within $10 \mathrm{De}$, the jet velocity of the central oxygen lance axis will reach a maximum value and then gradually decrease. As an explanation for this behavior, gas vortices are generated in the area surrounded by multiple jets near the top of the oxygen lance. The speed of the jet at the central axis of the oxygen lance will change when it passes through the gas vortex. When it enters the gas vortex area, the speed will decrease, and the speed will increase gradually after leaving this area.

3.4. Influence of Flow Rate on the Distribution of Radial Jet Parameters. The dynamic radial parameter distribution of the jet reflects the concentration of the jet [24]. The mechanical stirring effect of the jet on the molten pool is closely related to the dynamic pressure distribution of the oxygen jet on the cross section, which determines the impact area of the jet on the molten pool. By studying the radial dynamicpressure distribution of the jet, the jet characteristics can be better analyzed.

Figure 15 shows the radial dynamic-pressure distribution for oxygen lance positions between 20 De and 40 De for different oxygen lances. According to the results, the radial dynamic-pressure distribution law of each nozzle is similar. With the rise of the oxygen lance position, the radial dynamic pressure gradually declines; as the radial distance 


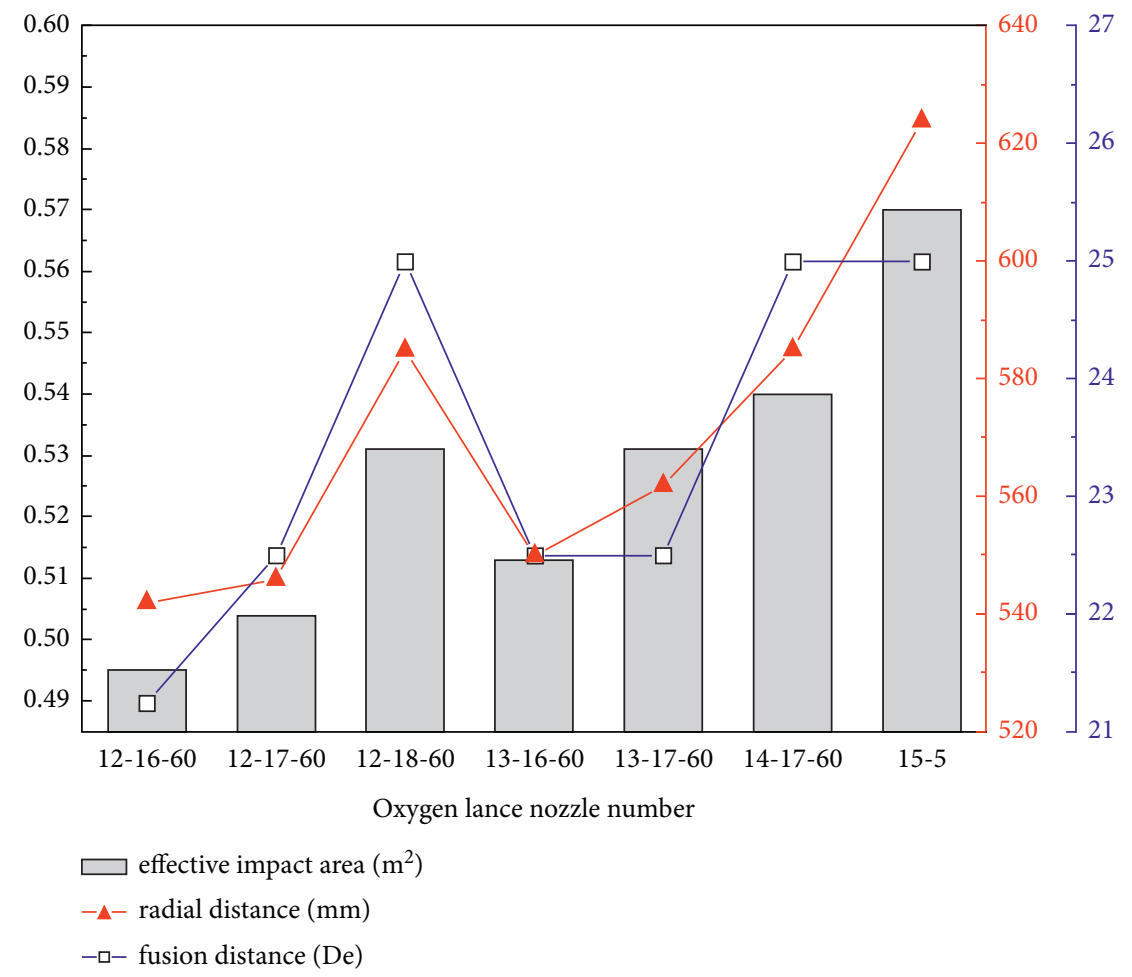

FIgURE 9: Variation of the effective impact area, fusion distance, and radial distance for different types of oxygen lances.

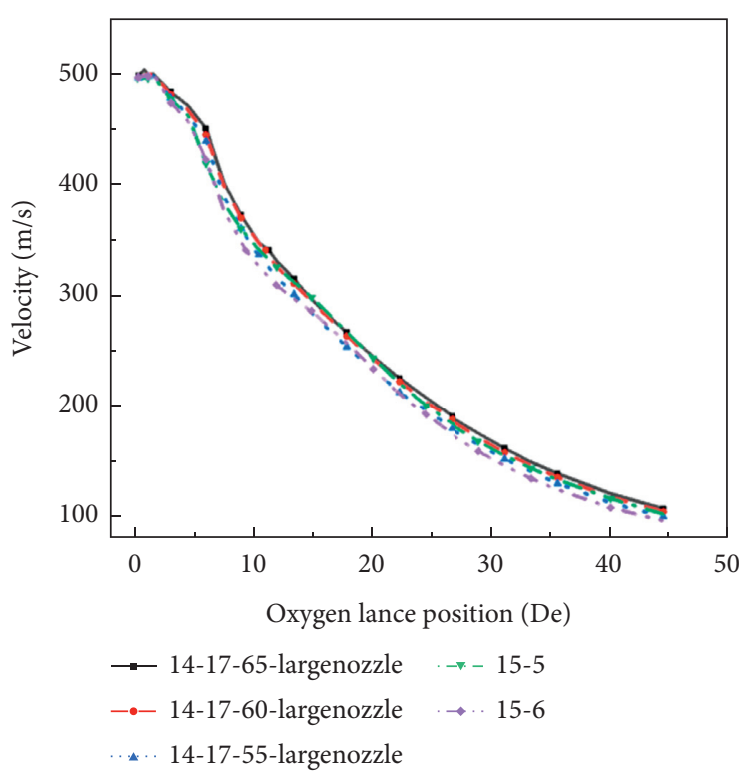

(a)

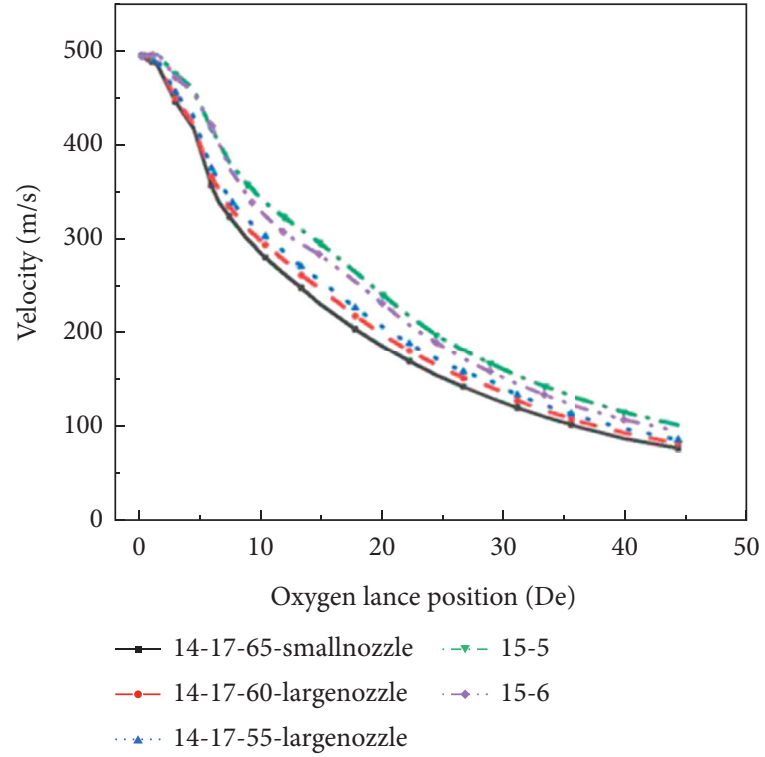

(b)

FIGURE 10: Axial velocity distribution along the central jet axis for the dual-structure oxygen lances and traditional oxygen lances. (a) Velocity distribution for large holes. (b) Velocity distribution for small holes.

increases, the radial dynamic-pressure distribution becomes flatter. Compared with the traditional oxygen lance, the radial dynamic pressure in the middle of the two jets of the dual-structure oxygen lance, that is, at the position of the geometric central axis of the oxygen lance nozzle, is significantly greater, and an increase in the oxygen lance position results in increased dynamic pressure. Therefore, concerning the dynamic radial parameter distribution of the oxygen lance, the dual-structure oxygen lance demonstrates advantages over the traditional oxygen lance. The jet of the 


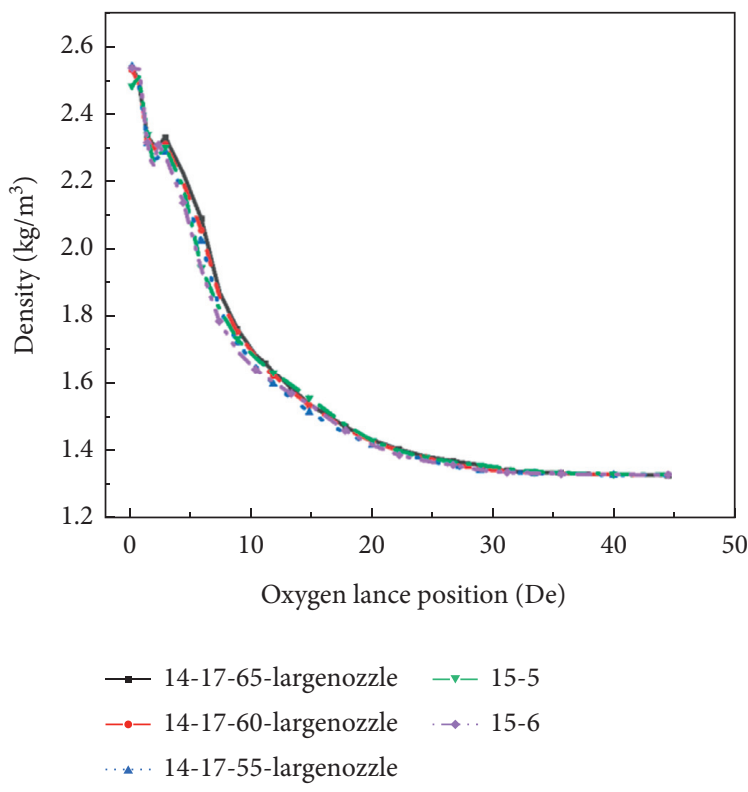

(a)

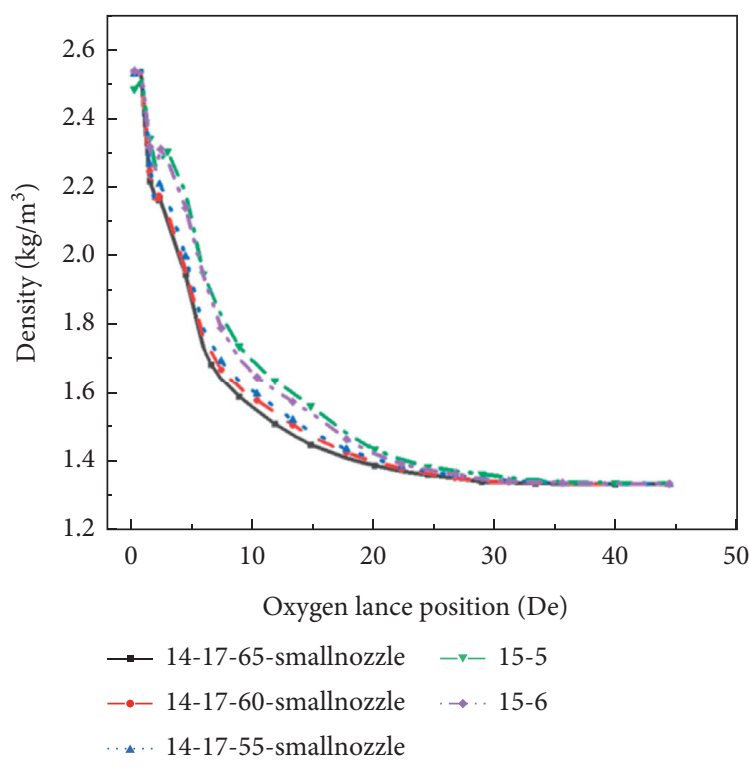

(b)

FIgURE 11: Axial density distribution along the central jet axis for the dual-structure oxygen lances and traditional oxygen lances. (a) Density distribution for large holes. (b) Density distribution for small holes.

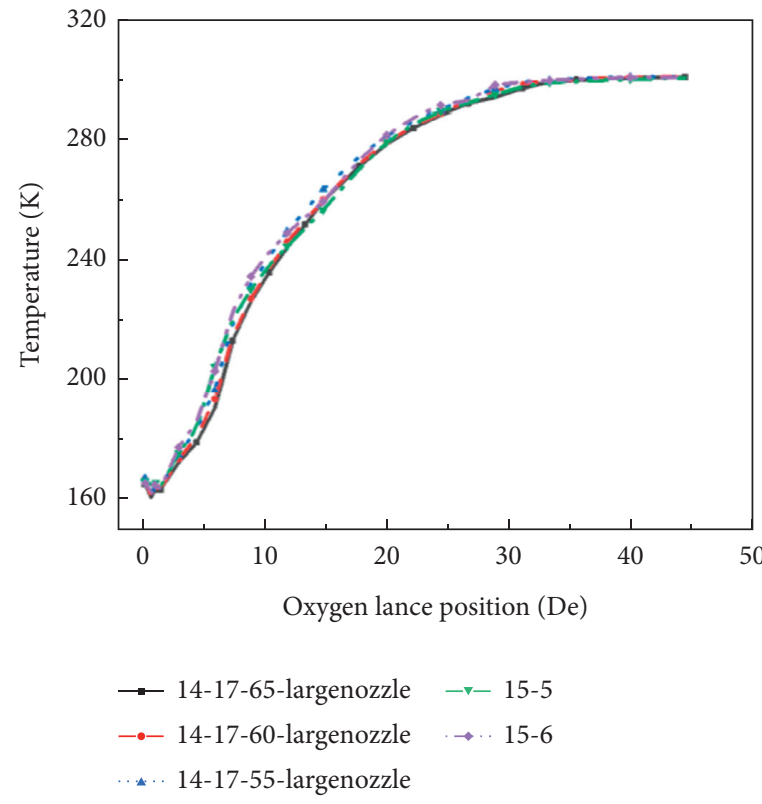

(a)

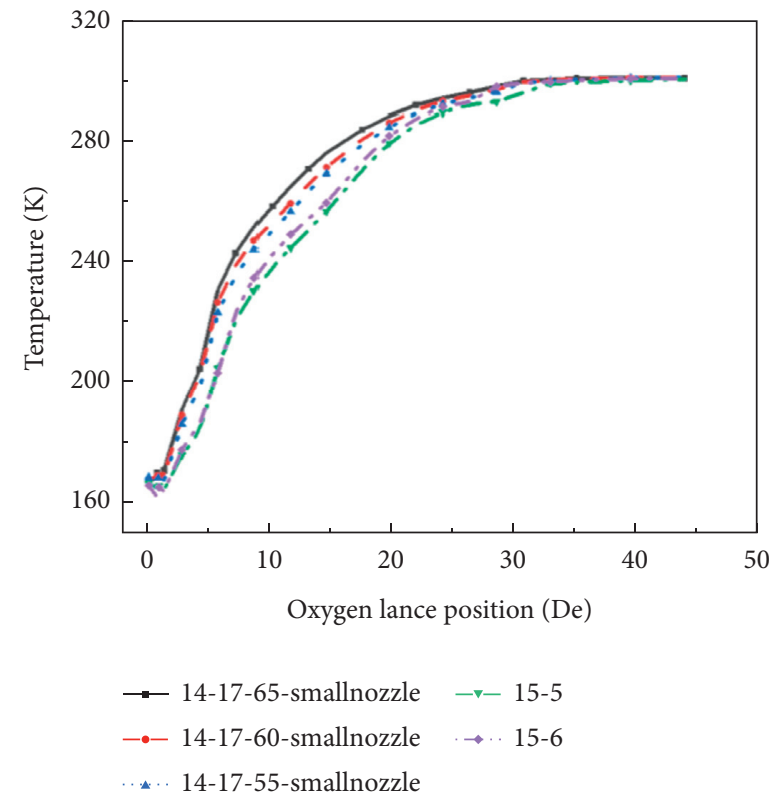

(b)

FIgURE 12: Axial temperature distribution along the central jet axis for the dual-structure oxygen lances and traditional oxygen lances. (a) Temperature distribution for large holes. (b) Temperature distribution for small holes.

new design exhibits greater momentum when impacting the molten pool, the depth of the pit formed by the impact is deeper, and the stirring of the molten pool is more uniform and powerful. In addition, the radial dynamic pressure at the middle position of the jet increases with the increase in the flow rate through the large holes in the dual-structure oxygen lance.
3.5. Influence of Flow Rate on the Jet Impact Area. The impact area is an important indicator to measure the interaction between the jet and the molten pool. It represents the jet impact characteristics of the supersonic oxygen lance and is different at different impact speeds. The impact area at an impact speed of 10,30 , and $70 \mathrm{~m} / \mathrm{s}$ is defined as $\mathrm{S} 10, \mathrm{~S} 30$, and S70, respectively. When the impact velocity reaching the 


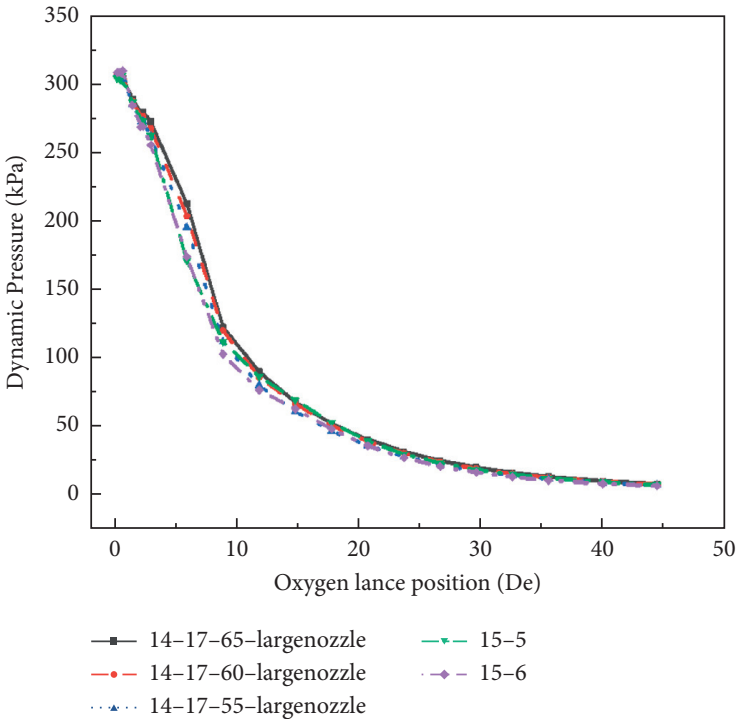

(a)

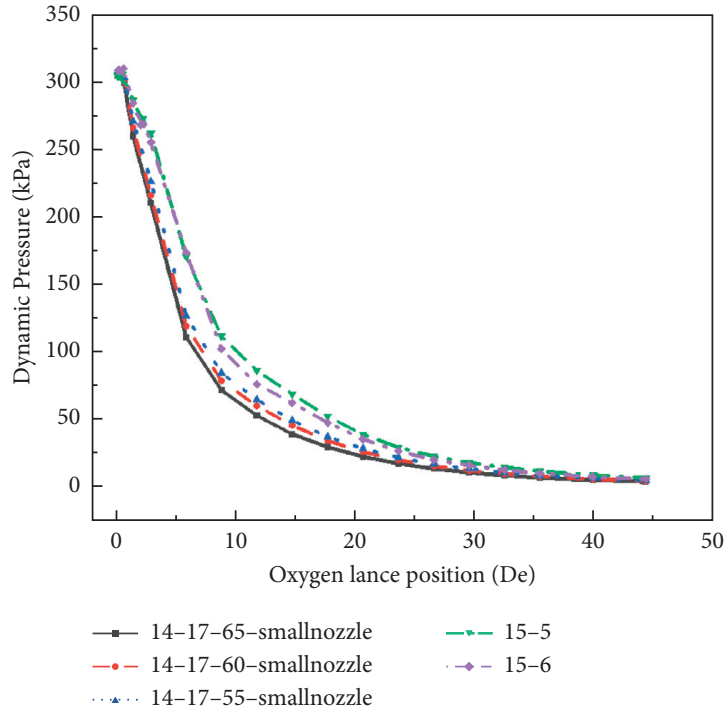

(b)

Figure 13: Axial dynamic pressure distribution along the jet central axis for the dual-structure oxygen lances and traditional oxygen lances. (a) Dynamic pressure distribution for large holes. (b) Dynamic pressure distribution for small holes.

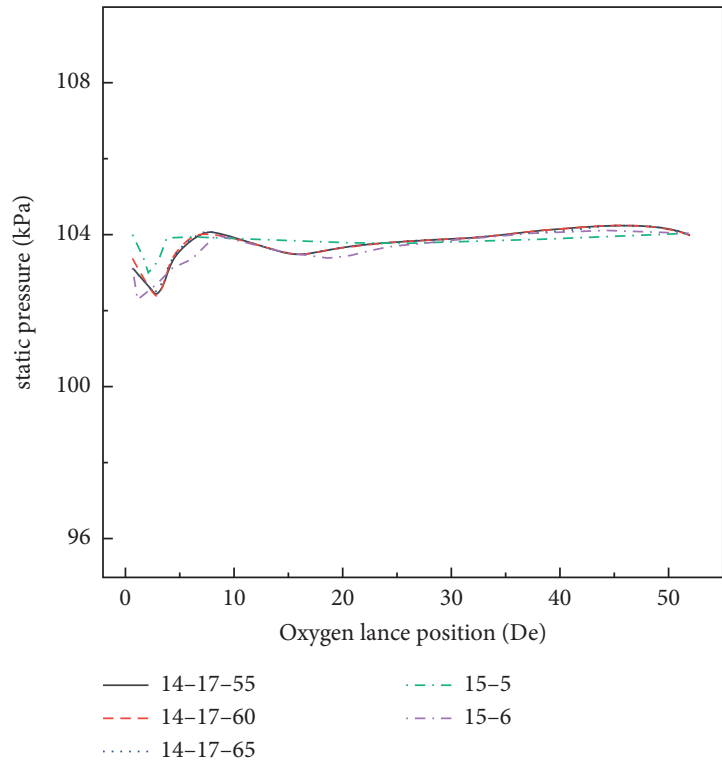

(a)

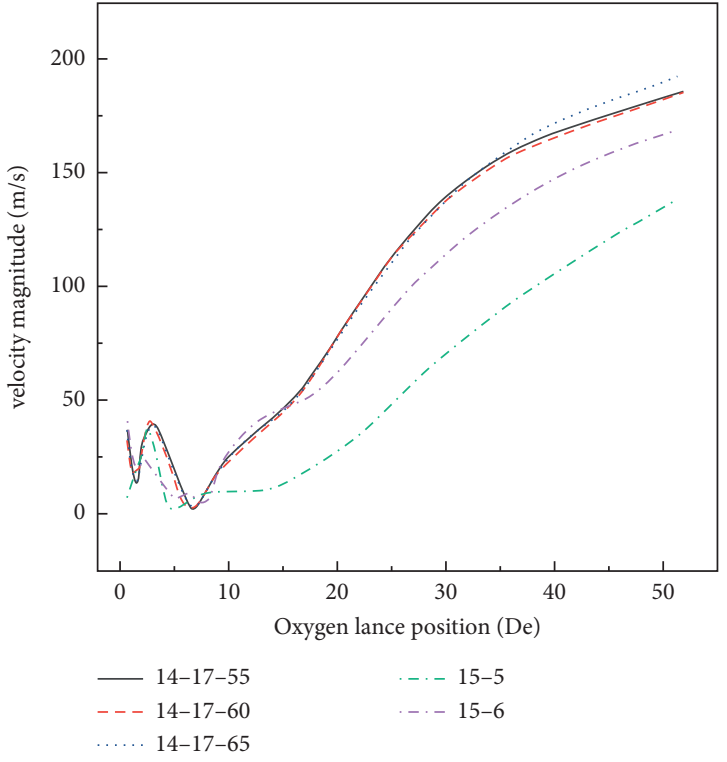

(b)

Figure 14: Variation of the velocity and static pressure on the geometric central axis of the different oxygen lance nozzles. (a) Variation of the static pressure. (b) Variation of the velocity.

molten pool is $10 \mathrm{~m} / \mathrm{s}$, the surface of the molten steel will produce some normal foaming slag, excessive foaming slag, and back-drying slag after reaction of the surface of the molten steel with oxygen. When the subsequent oxygen jet blows to the surface of the molten steel, these slag layers need to be broken first to contact the molten steel surface. When the impact velocity reaching the molten pool is $30 \mathrm{~m} / \mathrm{s}$, the jet will agitate the surface of the molten pool, and the jet's main contribution is the melting of the slag. At an impact velocity of $70 \mathrm{~m} / \mathrm{s}$, the jet can completely break through the slag layer and assumes the function of stirring whilst affecting mechanical agitation and chemical interaction of the molten steel.

The diagram in Figure 16 shows the comparison of the impact areas for the various oxygen lances at different impact velocities. The impact areas of the three dualstructure oxygen lances at low oxygen lance positions are similar and larger than the corresponding impact areas of 

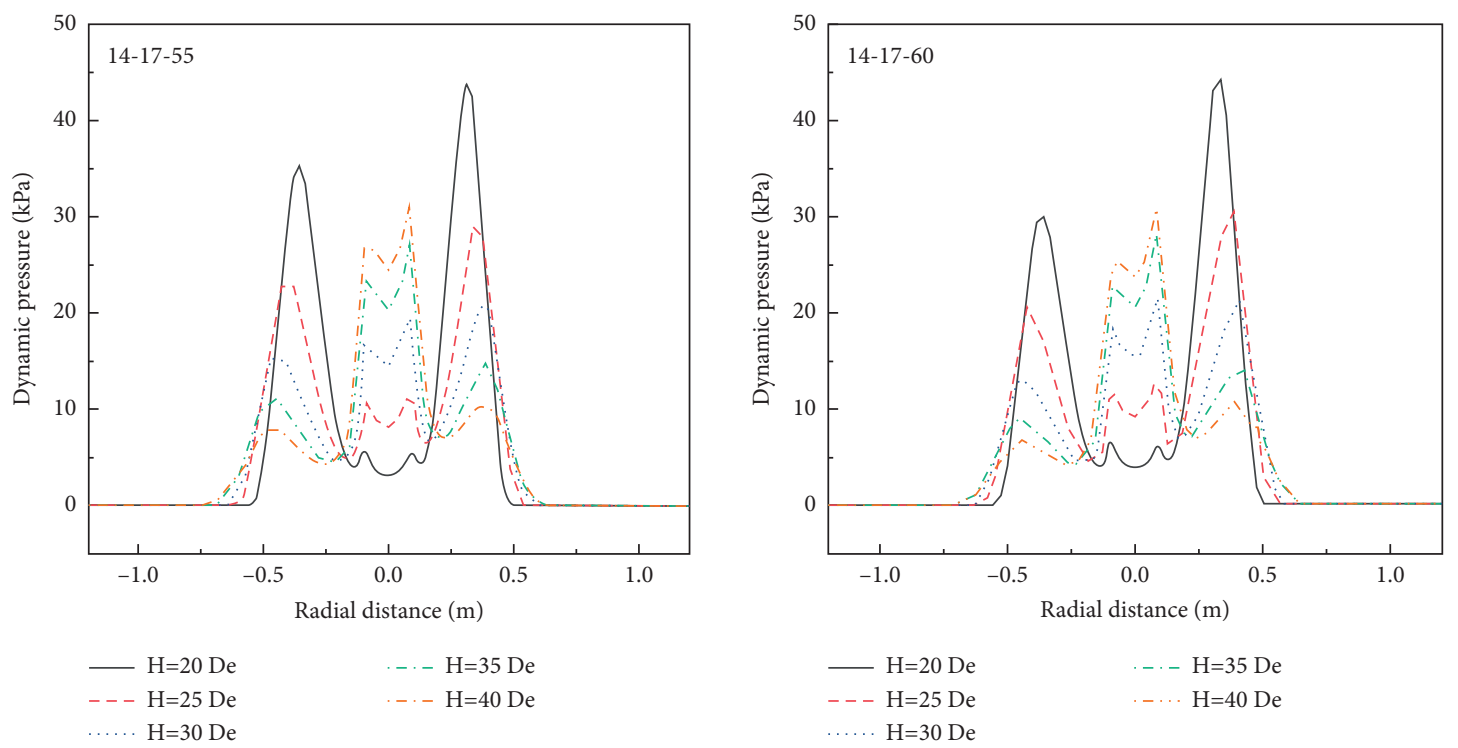

(a)
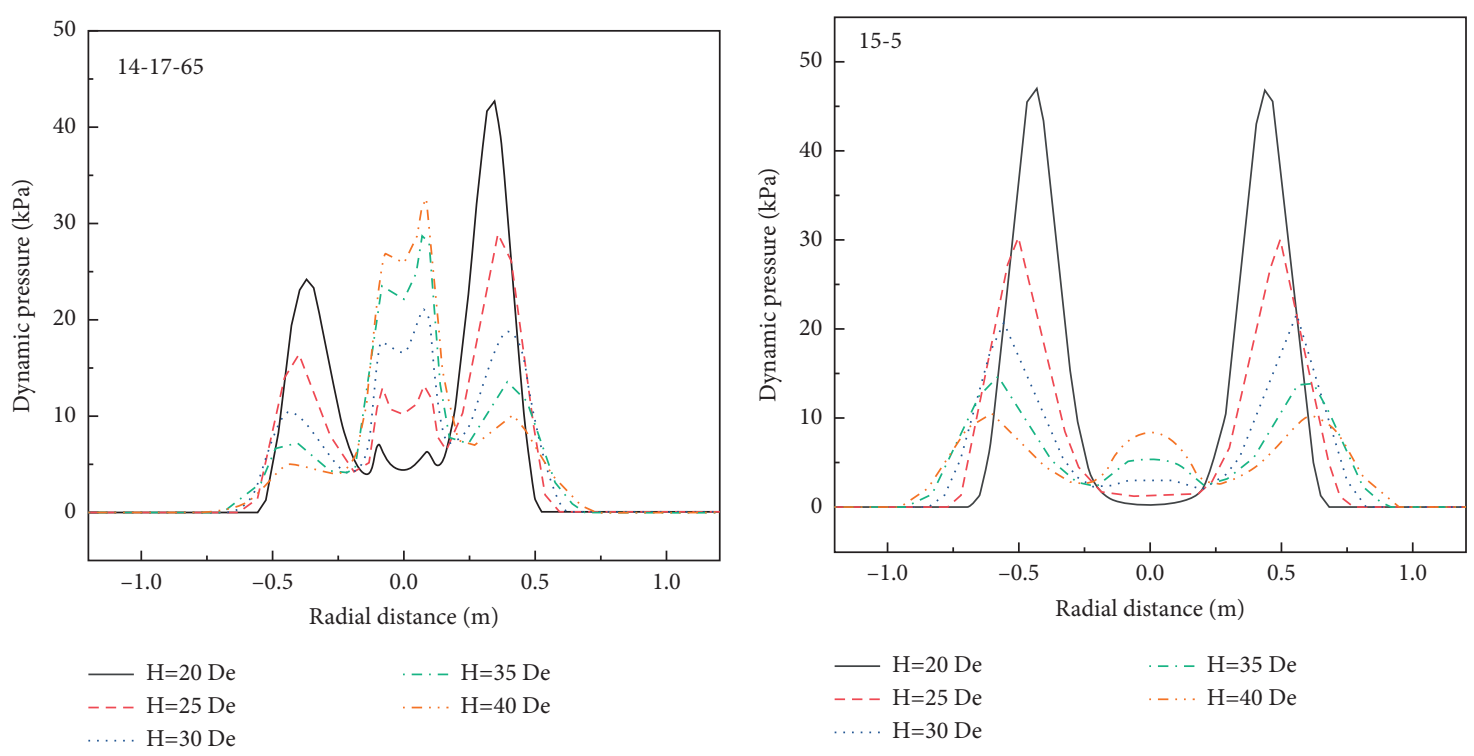

(c)

(d)

FIgURE 15: Continued. 


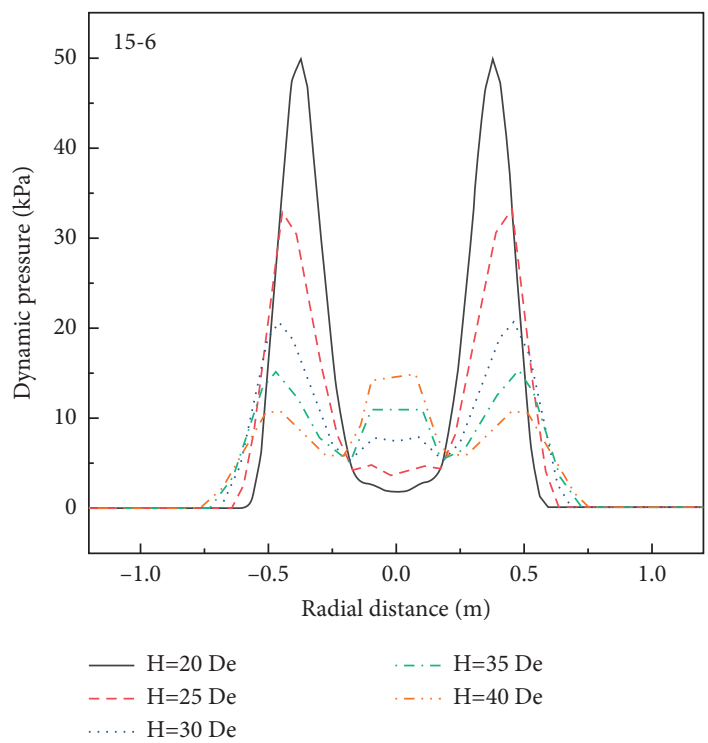

(e)

FIGURE 15: Radial dynamic-pressure distribution for different oxygen lance positions. (a) 14-17-55. (b) 14-17-60. (c) 14-17-65. (d) 15-5. (e) 15-6

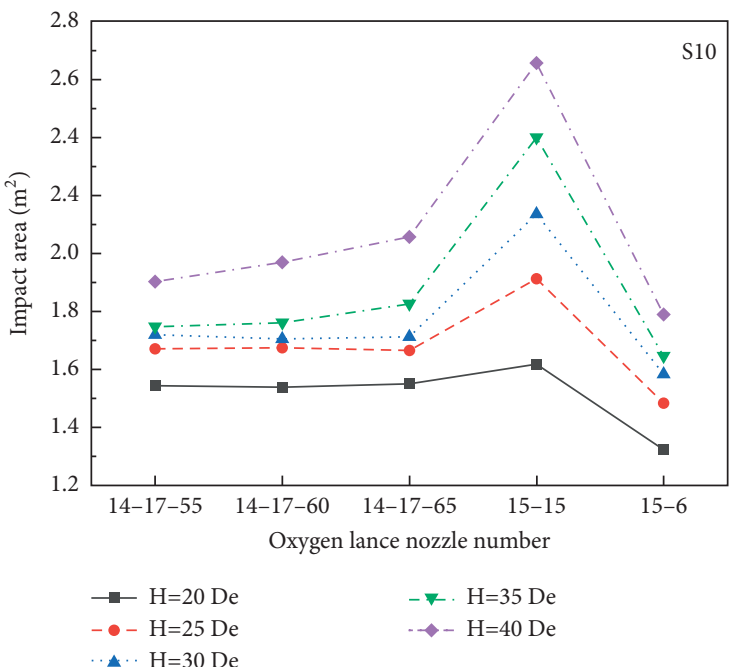

(a)

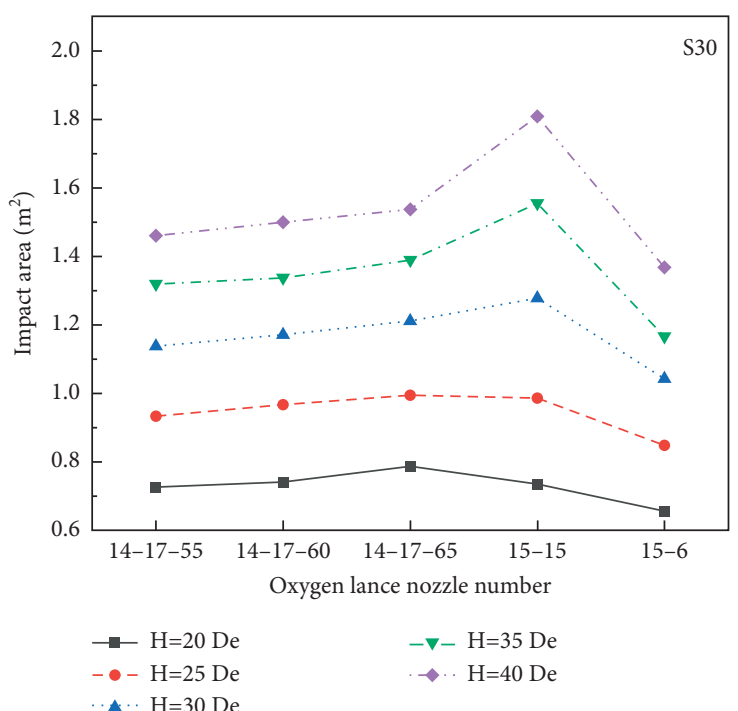

(b)

Figure 16: Continued. 


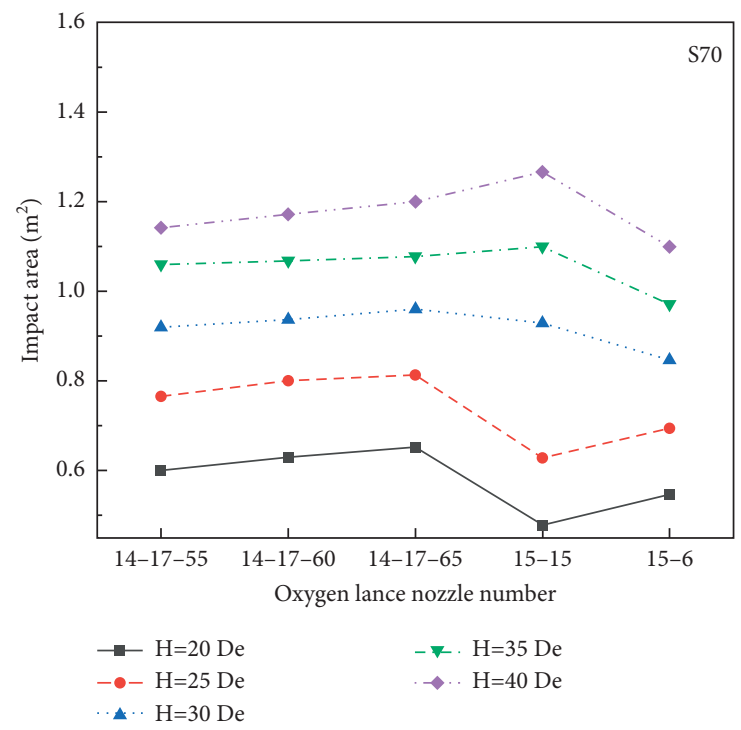

(c)

Figure 16: Impact area for the various oxygen lances at different impact velocities. (a) Impact velocity of $10 \mathrm{~m} / \mathrm{s}$. (b) Impact velocity of $30 \mathrm{~m} / \mathrm{s}$. (c) Impact velocity of $70 \mathrm{~m} / \mathrm{s}$.

the traditional six-hole oxygen lance. However, when the oxygen lance position is equal to or above $35 \mathrm{De}$, the impact area of lance 14-17-65 is larger than the other two lances. This difference is less obvious for higher impact velocities. It shows that the impact area of the dual-structure oxygen lance is significantly larger than that of the traditional sixhole oxygen lance, but the flow rate ratio has little effect on the impact area. As the oxygen lance position rises, the impact area gradually increases. The impact area of the traditional five-hole oxygen lance at the high oxygen lance position is larger than that of other oxygen lances. However, as the oxygen lance position descends, the advantage gradually decreases, and the impact area is smaller than that of the dual-structure oxygen lance at the impact velocity of $70 \mathrm{~m} / \mathrm{s}$. The results demonstrate that the jet of the dualstructure oxygen lance features a larger effective impact area and improved stirring of the molten pool.

\section{Conclusions}

(1) Multiple jets are deflected to the central axis of the oxygen lance during movement, and the inclination angle of the nozzle holes influences the deflection of the jet significantly. With smaller nozzle angles, the jet moves closer to the central axis of the oxygen lance, the radial distance of the jet decreases, and the mutual attraction between the streams increases.

(2) As the oxygen lance position rises, the impact area gradually increases. The impact area of the new dualstructure oxygen lance is significantly larger than that of the traditional six-hole oxygen lance, which is related to the independence of each jet. With larger nozzle angles, the jet independence improves, the fusion between the streams becomes slower, and the impact area increases. The flow rate ratio has little effect on the impact area.

(3) With the axial flow of the jet, the velocity, density, and dynamic pressure on the centerline of the jet gradually decrease, and the temperature of the jet steadily rises. The jet flow characteristics of the dual-structure oxygen lance are better than that of the traditional oxygen lance. The supersonic region is longer, and the negative pressure zone formed on the central axis of the oxygen lance is farther from the nozzle outlet. In the actual smelting process, the sticking of the oxygen lance can be effectively reduced and its service time increased.

(4) The dynamic radial parameter distribution of the dual-structure oxygen lance is superior to the traditional oxygen lance. Its jet impacts the molten pool with greater momentum, the depth of the pits formed by the impact is deeper, and the stirring of the molten pool is more uniform and powerful. Moreover, the radial dynamic pressure at the middle position of the jet increases with increasing the flow rate through the large holes in the dual-structure oxygen lance.

(5) A nozzle angle of $14^{\circ}$ combined with a flow rate ratio of $65 \%$ and a nozzle angle of $17^{\circ}$ combined with a flow rate ratio of $35 \%$ are the optimal parameters for the new dual-structure oxygen lance. Under these conditions, the length of both the supersonic region and the negative pressure region from the nozzle outlet is maximized. The largest impact area and the best stirring effect of the molten pool were found for oxygen lance positions beyond $35 \mathrm{De}$. 


\section{Data Availability}

The data used to support the findings of this study are included within the article.

\section{Conflicts of Interest}

The authors declare that there are no conflicts of interest regarding the publication of this paper.

\section{Acknowledgments}

The authors are grateful for the financial support by the National Natural Science Foundation of China (Grant no. NSFC52074151) and Education Department Project of Liaoning Province (Grant no. 2020LNQN12).

\section{References}

[1] M. Lv and R. Zhu, "Research on coherent jet oxygen lance in BOF steelmaking process," Metallurgical Research \& Technology, vol. 116, p. 13, 2019.

[2] J. H. Kwon, J. A. Lee, K. U. Lee, and J. W. A. Han, “A study on the supersonic jet nozzle to improve of the operating efficiency of the converter process," Korean Journal of Metals and Materials, vol. 58, p. 555, 2020.

[3] F. H. Liu, D. B. Sun, R. Zhu, and S. Y. Hu, "Effect of shrouding Mach number and ambient temperature on the flow field of coherent jet with shrouding Laval nozzle structure," Canadian Metallurgical Quarterly, vol. 58, p. 103, 2019.

[4] X. B. Zhou, M. Ersson, L. C. Zhong, J. K. Yu, and P. Jonsson, "Mathematical and physical simulation of a top blown converter," Steel Research International, vol. 85, p. 280, 2014.

[5] Z. F. Yuan, Y. Xiao, Z. X. Lu, J. N. Huang, and Y. F. Pan, "Jet behavior and metallurgical performance of innovated doubleparameter oxygen lance in BOF," Iron Steel Res, vol. 14, p. 3, 2007.

[6] W. Wang, Z. Yuan, H. Matsuura, H. Zhao, C. Dai, and F. Tsukihashi, "Three-dimensional compressible flow simulation of top-blown multiple jets in converter," ISIJ International, vol. 50, no. 4, pp. 491-500, 2010.

[7] M. Ersson, A. Tilliander, L. Jonsson, and P. Jönsson, “A mathematical model of an impinging air jet on a water surface," ISIJ International, vol. 48, no. 4, pp. 377-384, 2008.

[8] M. Ersson, L. Höglund, A. Tilliander, L. Jonsson, and P. Jönsson, "Dynamic coupling of computational fluid dynamics and thermodynamics software: applied on a top blown converter," ISIJ International, vol. 48, no. 2, pp. 147-153, 2008.

[9] L. L. Cao, Q. Liu, Z. Wang, and N. Li, "Interaction behaviour between top blown jet and molten steel during BOF steelmaking process," Ironmaking and Steelmaking, vol. 45, no. 3, pp. 239-248, 2018.

[10] A. Nordquist, M. Iguchi, S. Yokoya, L. Jonsson, and P. Jönsson, "A physical model study of swirl phenomena in a top blown bath," Steel Research International, vol. 77, no. 4, pp. 234-241, 2006.

[11] A. Nordquist, N. Kumbhat, L. Jonsson, and P. Jönsson, "The effect of nozzle diameter, lance height and flow rate on penetration depth in a top-blown water model," Steel Research International, vol. 77, no. 2, pp. 82-90, 2006.

[12] G. Liu, K. Liu, P. Han, and Y. Chen, "Numerical investigation on behaviors of interlaced jets and their interaction with bath in BOF steelmaking," AIP Advances, vol. 9, no. 7, p. 75202, 2019.

[13] X. Wang, G. Liu, and K. Liu, "Behaviors of supersonic doubleparameter jets and their impingement onto molten bath in BOF steelmaking," AIP Advances, vol. 10, no. 10, p. 105307, 2020.

[14] H. Jia, P. Han, K. Liu, Y. Li, K. Ba, and L. Feng, "Jet characteristics of a double-structure oxygen lance and its interaction with the molten pool in BOF steelmaking," AIP Advances, vol. 11, no. 8, p. 85330, 2021.

[15] C. He, N. Yang, and Q. Huang, "A multi-phase numerical simulation of a four-nozzle oxygen lance top-blown convertor," Procedia Earth and Planetary Science, vol. 2, p. 67, 2011.

[16] V. Panjkovic, J. Truelove, and O. Ostrovski, "Analysis of performance of an iron-bath reactor using computational fluid dynamics," Applied Mathematical Modelling, vol. 26, p. 211, 2002.

[17] M. Davis, K. Pericleous, M. Cross, and P. Schwarz, "Mathematical modelling tools for the optimisation of direct smelting processes," Applied Mathematical Modelling, vol. 22, p. 930, 1998.

[18] B. Liedmann, S. Wirtz, V. Scherer, and B. Krüger, "Numerical study on the influence of operational settings on refuse derived fuel co-firing in cement rotary kilns," Energy Procedia, vol. 120, p. 256, 2017.

[19] D. C. Wilcox, Turbulence Modeling for CFD, DCW Industries, Inc, La Cañada Flintridge, CA, USA, 1998.

[20] Q. Li, M. M. Li, S. B. Kuang, and Z. S. Zou, "Computational study on the behaviours of supersonic jets and their impingement onto molten liquid free surface in BOF steelmaking," Canadian Metallurgy Quarterly, vol. 53, p. 345, 2014.

[21] M. Lv, R. Zhu, Y. G. Guo, and Y. W. Wang, "Simulation of flow fluid in the BOF steelmaking process," Metallurgical and Materials Transactions B, vol. 44, p. 1567, 2013.

[22] I. Sumi, Y. Kishimoto, Y. Kikuchi, and H. Igarashi, "Effect of high-temperature field on supersonic oxygen jet behavior," ISIJ International, vol. 46, no. 9, pp. 1312-1317, 2006.

[23] R. Sambasivam and F. Durst, "Characteristics of supersonic jets in LD steelmaking," Ironmaking and Steelmaking, vol. 37, no. 3, pp. 195-203, 2010.

[24] M. Alam, J. Naser, and G. Brooks, "Computational fluid dynamics simulation of supersonic oxygen jet behavior at steelmaking temperature," Metallurgical and Materials Transactions B, vol. 41, no. 3, pp. 636-645, 2010. 